\title{
Utilização do instrumento de identificação de conhecimentos para administração segura de medicamentos com o uso de infusão automática
}

\author{
Léria Rosane Holsbach*, Francisco Jose Kliemann Neto, Nicole Holsbach
}

\begin{abstract}
Resumo Introdução: Entre os riscos intrínsecos na assistência à saúde, a infusão de medicamentos é uma preocupação constante. Essa pesquisa analisou os tipos de notificações de eventos adversos, com a possibilidade dos profissionais envolvidos no uso incorreto de operação de Bombas de Infusão (BI). Os autores realizaram uma pesquisa dos problemas decorrentes de eventos adversos gerados da utilização de BI e desenvolveram um instrumento de identificação de conhecimentos de habilidades e usabilidades de BI. Métodos: A metodologia utilizada no estudo foi desenvolvida em quatro fases. Na primeira e segunda fase, realizou-se uma pesquisa no banco de dados de relatos sobre notificações na unidade de Tecnovigilância da Agência Nacional de Vigilância Sanitária (ANVISA) com o levantamento de dados, classificação de equipamentos, tipificação de problemas e análise de causas comuns. Na terceira fase realizou-se a análise do banco de dados de notificações de um fabricante de BI. Na última fase foi feita a análise de causas comuns para desenvolver as ações preventivas e o desenvolvimento do instrumento proposto. Resultados: Foram analisados os anos de 2006 a 2011 onde existiam 1.971 relatos sobre notificações de equipamentos médicos hospitalares, sendo 251 desses relatos sobre BI (12,6\%). Essa porcentagem foi a maior encontrada entre todos os equipamentos notificados. Conclusão: O estudo permitiu afirmar que os profissionais que operam as BI, muitas vezes possuem carência de conhecimento e/ou falta de compromisso com a atividade. Pode-se afirmar que um aspecto importante e inquestionável sobre o uso de BI é a relação com a segurança.
\end{abstract}

Palavras-chave Bombas de infusão, Segurança do paciente, Eventos adversos, Usabilidade.

\section{Use of an instrument to identify knowledge for safe administration of medications using automatic infusion}

\begin{abstract}
Introduction: Among the inherent risks in health care, the infusion of drugs is a constant concern. This research examined the types of notification of adverse events, with the possibility of the health professionals involved in the incorrect use of Infusion Pumps (IP) operation. The authors conducted a research of the problems arising from adverse events generated using IP and developed an instrument to identify the skills and knowledge of BI usability. Methods: The methodology used in the study was developed in four phases. In the first and second phases, we carried out a search in the database of reports on notification in technovigilance unit of the Brazilian Health Surveillance Agency (ANVISA) with data collection, equipment classification, classification and analysis of problems of common causes. In the third phase took place the analysis of the database of notification from an IP manufacturer. In the last phase an analysis was made of the common causes for developing preventive actions and the development of the proposed instrument. Results: We analyzed the years 2006 to 2011 where there were 1.971 reports of medical equipment notification, and 251 of these reports on IP (12.6\%). This percentage was the highest among all equipment reported. Conclusion: The study allowed us to say that the professionals who operate the IP often denote lack of knowledge and / or lack of commitment to the activity. It can be argued that an important and unquestionable aspect about the use of IP is related to safety.
\end{abstract}

Keywords Infusion pumps, Patient safety, Adverse events, Usability. 


\section{Introdução}

O sistema de saúde configura um complexo de variedades de novas tecnologias e técnicas para atender a complexidade das patologias e a necessidade de novas alternativas de terapia, gerando inúmeros benefícios à população e ocasionando também novos riscos.

Entre os riscos intrínsecos na assistência à saúde a infusão de medicamentos é uma preocupação constante. A aplicação de medicamentos por via endovenosa é normalmente administrada em Bomba de Infusão (BI) (Rothschild et al., 2005). Qualquer erro de programação em uma bomba de infusão pode gerar graves consequências, podendo ser fatal dependendo do perfil do paciente ou da medicação utilizada.

Em torno de $80 \%$ dos pacientes hospitalizados recebem terapia por infusão. Com o aumento do uso de terapias intravenosas, tornou-se necessário desenvolver dispositivos para infundir drogas com pressão superior à pressão sanguínea e com precisão elevada (Button, 2002). Cerca de $30 \%$ dos danos durante a hospitalização estão relacionados a erros de medicação, os quais trazem também sérias consequências econômicas aos hospitais. O custo anual de danos severos a erros de medicação, nos USA, tem sido estimado em torno de U\$ 76,6 bilhões (Anderson et al., 2002; Berwick e Leape, 1999).

Uma pesquisa desenvolvida nos Estados Unidos da América (USA), em 36 unidades assistenciais de saúde, identificou como erros mais frequentes na administração de medicamentos: horário errado (34\%), dose errada (17\%) e aplicação de medicamento sem autorização (4\%) (Baker et al., 2002). Apesar de diferentes meios utilizados para a segurança na administração dos medicamentos, a frequência de uso e o potencial de risco de algum desses medicamentos tem levado a incidentes (Amoore e Adamson, 2003; Torelly, 2009). Os fatores humanos igualmente como a falta de conhecimento do principio de funcionamento, aplicação clinica e possíveis impactos na segurança desses equipamentos utilizados para administrar medicamentos podem contribuir para incidentes com possibilidade de fortes impactos negativos.

Nos Estados Unidos da América, as notificações sobre eventos adversos envolvendo equipamentos da área da saúde são compulsórias e podem ser relatados por qualquer cidadão norte americano. $\mathrm{O}$ órgão governamental que regula produtos para a saúde chama-se Food and Drugs Administration (FDA) e, no ano de 2010, recebeu 29.451 relatos de eventos adversos de BI, sendo 205 com graves consequências (Food..., 2010).

No Brasil, a vigilância pós-comercialização é competência da Tecnovigilância - unidade da Agencia Nacional de Vigilância Sanitária (Tecnovigilância - ANVISA), criada em 1999. Em 2001, foi implantada a Rede de Hospitais Sentinelas, que visava à criação de uma rede integrada atuando nas áreas de farmacovigilância, tecnovigilância e hemovigilância. No ano de 2004, foi lançada a Rede de Monitoramento de relatos de eventos adversos e, nesse mesmo ano, a Rede Nacional de Investigação de Surtos e Eventos Adversos (Lopes e Lopes, 2008) para incentivar os profissionais da saúde a notificar problemas com produtos da saúde. Nessa época, a ANVISA criou uma ferramenta chamada Sistema de Informação de Notificação de Eventos Adversos e Queixas Técnicas Relacionados a produtos de Saúde (SINEPS) para monitorar notificações envolvendo produtos sob a Vigilância Sanitária (Morita, 2009). A partir do ano de 2009, a ferramenta para monitorar as notificações passou a ser Notificações em Vigilância Sanitária (NOTIVISA).

Os relatos de notificações de tecnovigilância não eram compulsórios até o ano de 2010. A partir desse ano, com a criação da RDC 67, 2010 (Brasil, 2009), passaram a ser compulsórias. A consciência da importância de exercer a atividade de notificar está sendo lentamente desenvolvida no Brasil. A prática da notificação ainda está sendo compreendida.

Nos anos de 2006 a 2011, a Tecnovigilância recebeu 1971 relatos sobre queixas técnicas e eventos adversos de equipamentos. Os relatos notificados envolveram em torno de 98 tipos de equipamentos de diferentes tecnologias, sendo a maioria relatos sobre BI, os seja, do total foram 251 relatos de BI, $12,7 \%$.

A norma técnica NBR IEC 60601-2-24, 1999 - Parte 2: prescrições particulares para segurança de bombas e controladores de infusão - ABNT (Associação..., 1999) define que a utilização segura de bombas e controladores de infusão é primariamente de responsabilidade do operador.

A assistência à saúde é desenvolvida em ambientes dinâmicos e especializados onde ocorrem interações complexas entre doença, equipe, infraestrutura, equipamentos, processos, normas e procedimentos. Portanto, ações que exigem conhecimento técnico e científico ou habilidade técnica precisam ser realizadas em ambientes não planejados ou controlados (Guimarães, 2006; Runciman et al., 2002).

Essa pesquisa se propõe a realizar um estudo dos problemas decorrentes de eventos adversos gerados da utilização de BI e, com isso, desenvolver um instrumento de identificação de conhecimentos de habilidades e usabilidade.

Inicialmente a proposta era desenvolver um instrumento que pudesse ser aplicado a um amplo espectro de equipamentos médico hospitalares. Após 
a análise de 1.971 relatos de equipamentos do banco de dados sobre notificações de tecnovigilância sobre equipamentos médico hospitalares, da ANVISA, encontrou-se aproximadamente 98 tipos de diferentes tecnologias médicas, ou seja, equipamentos de princípio de funcionamento e aplicação clínica diferente. Assim sendo, foram encontradas dificuldades em desenvolver um modelo único para aplicar em diferentes tecnologias médicas.

Após a análise, optou-se por identificar relatos de equipamentos médicos hospitalares e ainda aqueles com maior porcentagem de relatos de notificações de tecnovigilância. $\mathrm{Na}$ análise encontrou-se que o maior número de problemas era relacionado com as bombas de infusão seguida de equipamentos de anestesia/ ventilação pulmonar, de oftalmologia, diálise e unidade eletrocirúrgica, ou seja, de principio de funcionamento e aplicação clinica diferente. Portanto, as habilidades para utilização desse tipo de equipamentos são de diferentes áreas de conhecimento (Schneider, 2002; Vidal e Carvalho, 2008; Wears e Perry, 2002; Zhang et al., 2003). Por esse motivo, encontrou-se dificuldade de desenvolver um instrumento único que atendesse de forma efetiva as diversas áreas de conhecimento. Diante do desafio exposto acima, o instrumento foi desenvolvido para o equipamento que apresentou o maior número de relatos encontrados, ou seja, as BIs.

\section{Algumas considerações sobre BI}

O sistema circulatório é o caminho primário para a oxigenação e nutrição do corpo humano e também para a remoção de dióxido de carbono e outras secreções do organismo.

Infusão significa introduzir um líquido (que não seja o sangue) em um vaso sanguíneo. Considerando que todo o sangue de um adulto saudável circula completamente em 60 segundos, as substâncias introduzidas no sistema circulatório são distribuídas rapidamente. Assim, rotas de acesso intravenoso e intra-arterial compõem vias eficazes para transportar fluidos, sangue e medicamentos até os órgãos vitais de um paciente.

As bombas de infusão foram desenvolvidas entre as décadas de 60 e 70 . A partir de então, estes dispositivos evoluíram conforme os avanços nos campos da eletrônica, da mecânica e da medicina.

Um sistema de infusão consiste tipicamente, de três componentes: um reservatório de fluido, um dispositivo (equipo) que transporta o líquido do reservatório para o paciente e um dispositivo para regular ou gerar o fluxo. Vários sistemas diferentes são usados para promover o fluxo de fluidos em equipos intravenosos. Uma bomba de infusão é um dispositivo eletromecânico capaz de gerar fluxo de um dado fluido a pressões superiores à pressão do sangue no local da infusão: cerca de $10 \mathrm{mmHg}$ para pressão venosa e aproximadamente $80 \mathrm{mmHg}$ e $120 \mathrm{mmHg}$ para pressão arterial diastólica e sistólica, respectivamente (Button, 2002). Possui alarmes e controles, possibilitando a infusão precisa e segura mesmo em baixas velocidades e longos períodos de tempo.

\section{Aplicações dos dispositivos de infusão}

Os dispositivos de infusão ambulatoriais ou de finalidade geral são usados para introduzir no sistema circulatório de pacientes líquidos e agentes farmacológicos através de rotas intravenosas (IV), epidurais e mais raramente intra-arteriais, em aplicações diversas como:

- A manutenção dos níveis apropriados de fluidos de um paciente durante e após cirurgias, tratamento de queimaduras e controle de desidratação em pacientes pediátricos;

- Nutrição parenteral (endovenosa) total (TPN) de pacientes;

- Para manter a veia aberta, facilitando a administração de medicamentos em emergência; (KVO - Keep Vein Open, é uma propriedade de bombas de infusão programáveis de redução do volume infundido no final da infusão, com o objetivo de manter o vaso aberto);

- Infusão contínua (por exemplo, hormônio do crescimento) ou intermitente (por exemplo, antibióticos) de drogas, em quantidades efetivas e não tóxicas.

A administração contínua de drogas pode ser considerada a aplicação mais importante dos dispositivos de infusão, pois a concentração de uma droga em seu sítio de ação deve ser suficientemente alta para ser efetiva, mas não tão elevada a ponto de ser tóxica. Os modos tradicionais de administração de drogas, através de injeções ou pílulas, resultam em flutuações ao longo do tempo na concentração da droga que podem fazer com que esta fique abaixo ou acima da faixa terapêutica, mesmo que a concentração média esteja dentro da faixa.

A infusão contínua de drogas reduz as flutuações e, se a taxa de infusão for correta, assegura uma ação terapêutica contínua. A infusão contínua de drogas pode ser usada para aplicação de: (i) drogas vasoativas, para controlar a pressão arterial; (ii) anestésicos durante cirurgias; (iii) quimioterapia para tratamento de câncer; (iv) agentes indutores de trabalho de parto; (v) drogas antiarrítmicas; (vi) insulina; (vii) supressores de dor e de trabalho de parto; (viii) hormônios e outros. 
Dispositivos de infusão são utilizados em pacientes hospitalizados em ambulatórios, centros cirúrgicos, unidades de terapia intensiva e em ambulâncias. Pacientes que necessitam de terapia prolongada de infusão, mas estão livres de outros cuidados hospitalares, podem ser tratados em casa.

\section{Sistemas de infusão}

Existem três tipos de sistemas de infusão: (i) o que utiliza controle manual de fluxo, e é mais simples de todos; (ii) o que utiliza um controlador de infusão (automático ou semiautomático) para estabelecer o fluxo determinado pelo operador; e (iii) a bomba de infusão, que gera, monitora e controla o fluxo (Button, 2002).

A bomba de infusão é sistema que oferece a maior precisão de infusão, e permite trabalhar com cessões maiores que os sistemas gravitacionais. Nas bombas de infusão o controle do fluxo pode ser volumétrico ou não-volumétrico. A pressão de infusão independe da pressão gravitacional, e muitas vezes é maior que a pressão gravitacional. O controle de fluxo pode ser volumétrico ou não volumétrico, e o mecanismo de infusão pode ser peristáltico, por pistão ou por seringa.

Nas BI existem vários sensores como sensor de ar, gotas, pressão (para detectar oclusão na linha) e transdutores de ultra som para medir o fluxo de infusão, final da infusão, reservatório vazio e erro de programação.

Também existem nas BI, um dispositivo que transporta o líquido do reservatório para o paciente, chamado equipo. $\mathrm{O}$ equipo pode ser específico (para determinada BI que está sendo utilizada) ou universal (para ser utilizada em BI peristáltica de maneira geral).

As BI são classificadas como de uso geral, de insulina, de infusão de analgésicos controlados pelo paciente, implantáveis e de múltiplos canais.

Os riscos mais comuns relacionados ao uso dos equipamentos são: fluxo livre que pode levar a sobre dose e ocorre normalmente devido ao posicionamento incorreto ou escape do equipo; erro de programação que pode levar a sobre dose ou sub dose; interferência eletromagnética que pode alterar a programação da BI; infecção ou necrose no local da infusão que pode ocorrer devido ao deslocamento da agulha ou até mesmo higienização inadequada.

\section{Engenharia dos fatores humanos}

No sistema de qualidade, o controle de projeto de um produto possui várias etapas. Dentre elas podemos citar: pesquisa clinica, gerenciamento de riscos e usabilidade até a transferência do projeto para a etapa de produção - ABNT (Associação..., 2009). $\mathrm{Na}$ a engenharia de fatores humanos os erros de diagnóstico e também de utilização de equipamentos médicos podem ser causados por atalhos cognitivos (heurísticos) que os profissionais tomam, muitas vezes em nome da eficiência. Por exemplo, muitos erros ocorrem quando existe pressa de fazer um julgamento ou uma atividade e depois precisam defender sua decisão vigorosamente quando surgem evidências contraditórias. Isso é da natureza humana, onde muitas vezes espera-se ver mais do que está na frente dos olhos (Watcher, 2010).

Uma das alternativas para redução de erros de utilização são os testes operacionais e a análise heurística onde procuram identificar equipamentos os sistemas propensos a falhas, antes que estas cheguem a causar danos. Nas avaliações heurísticas, a operacionalidade de um sistema particular ou equipamento é abordada pela aplicação de fundamento de desenho como visibilidade do status do sistema, controle e liberdade do usuário, consistência e padrões, flexibilidade e eficiência de utilização (Cybis, 2007; Gawande, 2007; Ginsburg, 2005; Kushnituk e Patel, 2004).

Durante uma avaliação heurística, os especialistas navegam na interface do usuário, procurando por aspectos de operacionalidade. Em essência, analistas tentam se colocar no lugar do usuário, procurando por situações de erro que possam comprometer a segurança do paciente. A principal falha da heurística, entretanto, é que ela é tão boa quanto o analista que está executando a avaliação. Sem colocar os usuários em situações reais e sem observar seu trabalho, torna-se difícil identificar aspectos problemáticos de projeto. Entretanto avaliações heurísticas podem fornecer informações importantes para a equipe de projetos ou até mesmo para o hospital que esteja utilizando ou adquirindo equipamentos (Aspden et al., 2007). Portanto, um dos objetivos é assegurar que esses equipamentos sejam projetados de forma a reduzir a probabilidade de erro e garantir a segurança.

O saber (conhecimentos adquiridos) são aspectos cognitivos e o fazer é a habilidade. O conhecimento são construções permanentes e não são inteiramente dependentes da tarefa a realizar (Guimarães, 2006). A ergonomia cognitiva tem a mobilização operatória das capacidades mentais do ser humano em situações de trabalho para resultar num bom manejo mental das coisas que a pessoa lida em seu trabalho e no seu cotidiano (Vidal e Carvalho, 2008). As habilidades estão associadas ao saber fazer, que se presume uma capacidade adquirida de realizar determinadas tarefas, específica para identificar variáveis, relacionar informações, analisar situações, julgar o que culmina na realização de uma tarefa (Lima, 2005; Saupe et al., 2005). A usabilidade pode ser compreendida como uma 
característica do fator humano relacionada à facilidade de uso, efetividade, eficiência e satisfação do usuário, e deve ser considerada desde o desenvolvimento do produto - ABNT (Associação..., 2011a, 2011b; Brasil, 2013). Mas deve-se lembrar que os seres humanos são falíveis e que com frequência superestimam suas habilidades e subestimam suas limitações. Para esse estudo, a contribuição foi a percepção do tópico de usabilidade dentro do controle do projeto.

\section{Métodos}

O estudo foi realizado em quatro fases (Tabela 1). A fase I (FI) e a fase II (FII) realizou-se pesquisa no banco de dados de relatos sobre notificações de equipamentos na Tecnovigilância da ANVISA. Foram analisados no banco de dados os anos de 2006 a 2011, onde existiam 1.971 relatos de equipamentos médicos hospitalares. Desses relatos, havia aproximadamente 98 tipos de diferentes tecnologias de equipamentos.

Inicialmente, após o levantamento de dados, identificaram-se os equipamentos com maior número de relatos de notificações onde foi realizada a análise, classificação e tipificação de problemas com a identificação de causas comuns para a preparação das ações de prevenção. Nas mesmas fases, identificou-se que o maior número de problemas era relacionado com as bombas de infusão seguida de equipamentos de anestesia/ventilação pulmonar, de oftalmologia, diálise e unidade eletrocirúrgica. Por razões descritas na justificativa optou-se a pesquisar BI. Foram identificados e analisados os relatos de 251 (12,7\%) de bombas de infusão nos 1.971 relatos.

A terceira fase (FIII) realizou-se uma pesquisa no banco de dados de notificações de eventos adversos de um fabricante de bombas de infusão nos anos de 2010 e 2011 coma a classificação, tipificação de problemas e identificação de causas comuns para a preparação de ações de prevenção.

A última fase (FIV) desenvolveu-se um instrumento de identificação de conhecimentos para habilidades e usabilidade de BI. O instrumento foi desenvolvido utilizando quatro conjuntos de informações: dados de notificações de eventos adversos oriundos $(i)$ da ANVISA (correspondentes a notificações recebidas pela unidade de Tecnovigilância nos anos de 2006 a 2011), e (ii) do fabricante de bombas de infusão (correspondentes a notificações recebidas nos anos de 2010 e 2011), além de conceitos teóricos de (iii) gerenciamento de risco e (iv) engenharia de fatores humanos e aspectos ergonômicos. $\mathrm{O}$ instrumento é composto por 23 questões, sendo uma questão, subdividida em partes (a) e (b), de natureza aberta, e 22 questões de natureza fechada. As informações em (i) foram utilizadas na elaboração das questões 2, 3, 6 a 12, e 14 a 15; as informações em (ii) serviram de base na elaboração das questões 1, 4, 4a e 20; as informações em (iii) foram utilizadas na elaboração das questões 5, 6, e 13; por fim, as informações em (iv) serviram de base para a elaboração das questões 16 a 19 e 21 a 23. A evolução das questões no instrumento explora os seguintes aspectos de interesse na pesquisa sobre

Tabela 1. Quadro de fases/etapas/passos do projeto. Table 1. Phases/stages/steps of the project.

\begin{tabular}{|c|c|c|}
\hline Fase & Etapas & Passos \\
\hline I & $\begin{array}{l}\text { E1 - Pesquisa no banco de dados de relatos } \\
\text { de equipamentos médico hospitalares/ } \\
\text { eletromédicos sobre notificações na } \\
\text { Tecnovigilância da Agência Nacional de } \\
\text { Vigilância Sanitária - ANVISA. }\end{array}$ & $\begin{array}{l}\text { P1 - Analisados no banco de dados relatos de equipamentos } \\
\text { dos anos de } 2006 \text { a } 2011 \text {. } \\
\text { P2 - Levantamento dos dados. } \\
\text { P3 - Classificação do tipo de equipamento. } \\
\text { P4 - Identificação de equipamentos com maior porcentagem de } \\
\text { relatos de eventos adversos e queixas técnicas. }\end{array}$ \\
\hline II & $\begin{array}{l}\text { E2 - Pesquisa no banco de dados de relatos } \\
\text { de equipamentos médico hospitalares/ } \\
\text { eletromédicos sobre notificações na ANVISA } \\
\text { com o maior porcentagem de eventos adversos. }\end{array}$ & $\begin{array}{l}\text { P1 - Identificação do equipamento com maior porcentagem de } \\
\text { relatos de eventos adversos e queixas técnicas. } \\
\text { P2 - Analisados no banco de dados } 251 \text { relatos de BI. } \\
\text { P3 - Tipificação dos problemas. } \\
\text { P4 - Classificação dos problemas. } \\
\text { P5 - Identificação das causas comuns. } \\
\text { P6 - Preparação das ações de prevenção. }\end{array}$ \\
\hline III & $\begin{array}{l}\text { E3 - Pesquisa no banco de dados de } \\
\text { notificações de eventos adversos de um } \\
\text { fabricante de BI. }\end{array}$ & $\begin{array}{l}\text { P1 - Análise dos relatos de eventos adversos do ano de } 2010 \text { e } \\
2011 . \\
\text { P2 - Tipificação dos problemas. } \\
\text { P3 - Identificação das causas comuns. } \\
\text { P4 - Preparação das ações de prevenção. }\end{array}$ \\
\hline IV & $\begin{array}{l}\text { E4 - Desenvolvimento de um instrumento de } \\
\text { identificação de conhecimentos para habilidades } \\
\text { e usabilidades. }\end{array}$ & P1 - Disponibilizar para ser utilizado. \\
\hline
\end{tabular}


bombas de infusão: habilidades necessárias à operação do equipamento, conhecimento sobre gerenciamento de riscos e eventos adversos relacionados à utilização do equipamento, bem como sua frequência percebida de ocorrência, aderência do usuário do equipamento a procedimentos operacionais e adequação dos aspectos ergonômicos e engenharia de fatores humanos à tarefa a ser desenvolvida.

\section{Resultados}

Inicialmente o estudo possibilitou constatar que, no banco de dados da ANVISA, o sistema para gerenciar riscos utilizados permitiu distinguir relatos de ocorrências envolvendo produtos para a saúde sob vigilância sanitária (os dados analisados foram somente relatos envolvendo equipamentos médico hospitalares). Foi possível uma análise inicial do cenário, onde identificou-se problemas com BI, mas não pode-se precisar a porcentagem de utilização inadequada desse equipamento.

Foram analisados no banco de dados dos anos de 2006 a 2011 aproximadamente 1.971 relatos de notificações de tecnovigilância equipamentos médicos hospitalares. O banco de dados foi disponibilizado pela ANVISA e analisado em três períodos, conforme mostrado abaixo. Desses relatos, foram identificados problemas de 251 bombas de infusão, ou seja, 12,6\% foram problemas ocorridos em BI. Essa porcentagem foi à maior encontrada de todos os equipamentos notificados no banco de dados analisado.

Os relatos na Tecnovigilância - ANVISA estavam agrupados em três períodos: ano de 2006 a 2009; ano de 2010 e ano de 2011. Nessa pesquisa, os períodos foram analisados de forma a respeitar os critérios criados pela Tecnovigilância descritos a seguir.

\section{Período 2006-2009}

Foram analisados relatos voluntários de notificações de tecnovigilância de BI do ano de 2006 ao ano de 2009 Nesse período, houve 759 relatos de equipamentos médicos hospitalares/eletromédicos e 111 relatos foram sobre BI. Dos relatos de todos os equipamentos desse período, em torno de $14,6 \%$ foram de BI.

Os maiores problemas relatados foram apresentados na seguinte ordem: sensor de gotas $30(27,0 \%)$, comando trancado $22(19,8 \%)$, infusão20 (18,0\%), alarmes $15(13,5 \%)$ e relacionados a equipo e intervenções preventivas e calibrações 11 (9,9\%).

Nesse período analisado, os relatos foram de ordem confusa e com poucas informações, porém entendeu-se ser o inicio do exercício no Brasil de realizar a prática de notificar eventos adversos com equipamentos na área da saúde.

\section{Período 2010}

Analisaram-se os relatos de notificações de tecnovigilância envolvendo BI do ano de 2010. Nesse período, houve 299 relatos de equipamentos médico hospitalares e $16(5,3 \%)$ relatos foram relacionados a BI. Os problemas mais relatados foram com infusão e vazamentos.

Os problemas apresentados foram: infusão 7 (\%), vazamentos $4(\%)$, alarmes e problemas com roldana 2 (\%). Apesar do pequeno número de relatos de notificações sobre equipamentos médicos, percebeu-se que a causa mais relatada nesse período foi também relatada no primeiro período analisado, problemas com infusão (quase a metade do total dos relatos). Do mesmo modo, não foi possível identificar falhas relacionadas ao uso de $\mathrm{BI}$.

\section{Período 2011}

Analisaram-se os relatos de notificações de tecnovigilância envolvendo BI do ano de 2011. Nesse período, houve 913 relatos de equipamentos médico hospitalares e $124(13,6 \%)$ relatos foram sobre BI, sendo que um relato envolveu 4 BI. Nesse período, os maiores problemas encontrados foram com infusão (69 relatos - 55,6\%), alarmes (23 relatos $-18,5 \%$ ), sensor (sujo) com 9 relatos $(7,25 \%$ ) e BI não funciona com 8 relatos $(6,45 \%)$.

$\mathrm{Na}$ Tabela 2 são apresentados os relatos de notificações de tecnovigilância envolvendo BI no ano de 2006 a 2011 - Brasil.

\section{Análise do banco de dados de um fabricante de BI do ano de 2011}

Com o objetivo de obter mais uma comparação e ratificação dos dados encontrados nas análises anteriores, buscou-se o banco de dados de notificações de um fabricante de BI com análise de relatos de notificações recebidas pelo mesmo.

Nessa análise, pode-se identificar com precisão a porcentagem de problemas causados somente pela utilização inadequada desse equipamento. Os problemas mais comuns encontrados foram: cabo de força sem o pino terra, sensores danificados, sensores identificados com problemas, porém, ao serem avaliados, estavam em perfeito estado de uso, sensores inoperante devido a falta de limpeza, partes das bombas de infusão danificados (teclado, fonte de alimentação, gabinetes), partes faltantes (apoio da BI) e bateria descarregada. Os defeitos de utilização em 2010 foram em torno de 30\%, porém, com a ação de educação continuada realizada nos hospitais pelo próprio fabricante, onde utilizava a $\mathrm{BI}$, o índice de erros foi reduzido a 24\% em 2011, conforme Tabela 3. 


\section{Discussão}

Pelos resultados apresentados pode-se constatar que as respostas obtidas na análise do banco de dados da Tecnovigilância - ANVISA do ano de 2006 ao ano 2011 apresentaram dificuldades em classificar a tipificação do erro, se era relacionada ao uso da BI ou da falha do produto. A análise permitiu afirmar que tipos de erros encontrados relacionados à utilização incorreta das bombas de infusão são os relatos de sensor com sujeira e cabo desconectado. Do mesmo modo, os problemas relacionados com infusão, vazamentos, equipos e alarmes não permitiram afirmar o tipo do erro, podendo ser do modo de utilização incorreto realizado pelo usuário ou problemas técnicos do produto. As causas relatadas como falta de manutenções preventivas ou calibrações não foi possível compreender o significado da notificação.

Nesse mesmo banco de dados, no ano de 2010 também não foi possível identificar com precisão a causa do reduzido número de relatos de eventos adversos dos equipamentos na Tecnovigilância. Na tentativa de resgatar a memória da Tecnovigilância da ANVISA nesse ano, houve a informação da troca do sistema da tecnologia da informação (TI) e provavelmente o banco de dados foi corrompido. Inicialmente era utilizado o sistema de informatização denominado Sistema de Informação de Notificação de Eventos Adversos e queixas técnicas Relacionadas a produto para a Saúde (SINEPS) e atualmente denominado Sistema de Notificações em Vigilância Sanitária (NOTIVISA).

Na última análise do banco de dados do fabricante de bombas de infusão, foi possível avaliar e identificar os erros de utilização e, assim, comprovar a necessidade de conscientização dos profissionais sobre os danos causados ao paciente pela falta de conhecer o produto e seus impactos do que está sendo utilizando. A análise permitiu também verificar que os problemas encontrados tinham causas comuns nos bancos de dados estudados e possibilitou a preparação das ações preventivas para a segurança do paciente.

Tabela 2. Relatos de notificações de tecnovigilância envolvendo BI nos anos de 2006 a 2011 - Brasil. Table 2. Reports of technovigilance notifications involving BI in the years of 2006 to 2011 - Brazil.

\begin{tabular}{|c|c|c|c|c|}
\hline Relato & $\begin{array}{c}\mathrm{N}^{\circ} \text { de relatos } \\
2006-2009\end{array}$ & $\begin{array}{c}\mathrm{N}^{\circ} \text { de relatos } \\
2010\end{array}$ & $\begin{array}{c}\mathrm{N}^{\circ} \text { de relatos } \\
2011\end{array}$ & $\begin{array}{c}\text { Possíveis eventos } \\
\text { adversos }\end{array}$ \\
\hline Infusão. & 20 & 7 & 69 & \multirow{18}{*}{$\begin{array}{c}\text { Óbito; } \\
\text { Dano temporário; } \\
\text { Dano temporário } \\
\text { com prolongação } \\
\text { hospitalar; } \\
\text { Dano permanente. }\end{array}$} \\
\hline Alarmes. & 15 & 2 & 23 & \\
\hline Sensor (sujo). & 30 & - & 9 & \\
\hline Não funciona. & - & - & 8 & \\
\hline Vazamentos. & - & 4 & 3 & \\
\hline Interferência em outros equipamentos. & - & - & 3 & \\
\hline Rolamentos. & - & - & 3 & \\
\hline Equipo. & 11 & - & 2 & \\
\hline Câmara com corpo estranho. & - & - & 1 & \\
\hline Seringa. & - & - & 1 & \\
\hline Choque. & - & - & 1 & \\
\hline Cabo desconectado. & - & - & 1 & \\
\hline Comando travado. & 22 & - & - & \\
\hline Falta de calibração e/ou preventiva. & 11 & - & - & \\
\hline Bateria. & 1 & - & - & \\
\hline Desliga espontaneamente. & 1 & - & - & \\
\hline Problemas de roldana. & - & 2 & - & \\
\hline Travamento de botões. & - & 1 & - & \\
\hline
\end{tabular}

Tabela 3. Relatos de eventos adversos de BI no ano de 2010/2011.

Table 3. Reports of BI adverse events during the year 2010/2011.

\begin{tabular}{|c|c|c|c|}
\hline \multirow{2}{*}{ Relato } & \multicolumn{2}{|c|}{$\%$ geral } & \multirow{2}{*}{ Possíveis eventos adversos } \\
\hline & 2010 & 2011 & \\
\hline $\begin{array}{l}\text { Cabo de força danificado. } \\
\text { Sensor danificado. } \\
\text { Sensores com sujeiras. } \\
\text { Bateria descarregada. }\end{array}$ & $30 \%$ & $24 \%$ & $\begin{array}{l}\text { Óbito; } \\
\text { Dano temporário; } \\
\text { Dano temporário com prolongação hospitalar; } \\
\text { Dano permanente. }\end{array}$ \\
\hline
\end{tabular}




\section{Instrumento de Identificação de Conhecimentos para Habilidades e Usabilidade de Bombas de Infusão - Recomendações Gerais para Utilização Segura da BI}

1- Assinale com um $\mathrm{x}$ a resposta que ao seu entender melhor descreve um acidente:

( ) Inevitável.

( ) Evitável com melhor tecnologia.

( ) Evitável com outra técnica.

( ) Evitável com melhor treinamento.

2- Você recebeu treinamento para utilizar a Bomba de Infusão (BI)?

( ) Sim. Quem aplicou o treinamento

( ) O treinamento foi repetido? ( ) Sim ( ) Não

Quanto tempo

3- Houve algum tipo de avaliação após treinamento?

4- Explique como você faz para utilizar uma BI.

4 a - Qual é a parte que você tem mais dificuldade para realizar essa ação?

5- Em caso de falha do equipamento, você conhece a severidade do dano?

( ) Sim.

( ) Não.

6 - Marque com um $\mathrm{x}$ os perigos potenciais sobre BI que você tem conhecimento:

( ) Óbito.

( ) Dano temporário.

( ) Dano temporário com prolongação hospitalar.

( ) Dano permanente.

7 - Quais as situações de risco que já ocorreram durante a utilização da BI onde você trabalha:

( ) Superdosagem.

( ) Subdosagem.

( ) A infusão correu em tempo maior.

( ) A infusão correu em tempo menor.

( ) Não infundiu.

( ) Local da aplicação da agulha sem assepsia.

( ) Agulha fora do local correto da aplicação.

( ) interpretação incorreta da prescrição médica.

8 - Se ocorreu erro de utilização que você conhece marque com um x:

( ) Equipo inadequado.

( ) Ajuste incorreto do equipo.

( ) Oclusão no sistema de infusão.

( ) Carga fraca da bateria.

( ) Existência de vazamentos.

( ) Falha no sistema de alimentação elétrica.

( ) agulha fora do local correto da aplicação.

( ) Configuração incorreta do equipo.

( ) Bateria descarregada.

( ) Presença de sujeira no sensor.

( ) Sensor com defeito.

( ) Erro de conversão entre unidades de medidas.

9 - Ao utilizar o equipamento você verifica o prazo de validade da manutenção preventiva? ( ) Sim. ( ) Não.

10 - Qual a frequência que você utiliza a BI? ( ) Diária. ( ) Semanal. ( ) Mensal.

11 - Você verifica a densidade do liquido? ( ) Sim. ( ) Não.

12 - Você verifica a carga da bateria? ( ) Sim. ( ) Não.

13 - Você faz a verificação prévia dos medicamentos a ser aplicado? ( ) Sim. ( ) Não.

14 - Você faz a verificação prévia da programação da BI? ( ) Sim. ( ) Não.

15 - Você faz a inspeção visual (cabo, sensor, painel e outros) prévia a da BI? ( ) Sim. ( ) Não.

16 - O barulho de seu local de seu setor (ou fora) interfere no seu trabalho? ( ) Sim. ( ) Não.

17-A iluminação de seu local de seu setor (ou fora) interfere no seu trabalho? ( ) Sim. ( ) Não.

18 - A temperatura de seu local de seu setor (ou fora) interfere no seu trabalho? ( ) Sim. ( ) Não.

19 - Você costuma trabalhar mesmo fazendo uso de algum medicamento, por exemplo: medicação para dor de cabeça, resfriado e outros? ( ) Sim. ( ) não

20 - Os manuais estão disponíveis para você consultar, caso necessite?

21 - A linguagem utilizada na BI é familiar para você?

22 - Quando você utiliza a BI você se considera no controle?

23 - As instruções do teclado da BI é suficiente para você utilizar o equipamento?

Figura 1. Instrumento de identificação de conhecimentos para habilidades e usabilidades de bombas de infusão.

Figure 1. Instrument for the identification of knowledge of skills and usability of infusion pumps. 
Observa-se que houveram algumas limitações metodológicas por conta da necessidade de sigilo de alguns dados.

De forma geral, o estudo analisou tipos de notificações de eventos adversos com a possibilidade dos profissionais envolvidos no uso incorreto de operação de bombas de infusão. Foram pesquisados três bancos de dados de notificações de eventos adversos entre os anos de 2006 a 2011 e pode-se confirmar a utilização de forma inadequada de BI. $\mathrm{O}$ instrumento proposto na Figura 1 foi gerado a partir da análise dos eventos adversos do material estudado, dentre os principais tópicos abordados podem-se citar: segurança, acidentes, capacitação, utilização, riscos, falhas.

Desse modo, é possível afirmar que os profissionais que operam as bombas de infusão muitas vezes denotam carência de conhecimento e/ou a falta de compromisso com a atividade. Ou não receberam o treinamento adequado ou ainda negligenciaram as informações recebidas, o que aumenta as complicações na terapia do paciente, principalmente na administração automática de soluções intravenosas em doses diferentes do prescrito.

Pode-se afirmar que um aspecto importante e inquestionável sobre o uso de bombas de infusão é a relação com a segurança. Se por um lado elas permitem uma precisão no tempo, volume e fluxo, por outro lado, elas têm sido descritas, na literatura da área, como potencialmente relacionadas a erros de medicação. Esse risco não é distribuído igualmente, alguns pacientes podem sofrer eventos significativos com fortes impactos e outros eventos menos significativos como hospitalização prolongada. Por exemplo, pacientes com uso de múltiplos medicamentos ou em uso de medicamentos de alto risco tem uma probabilidade maior de sofrer o risco. Esses riscos são resultados da interação de pessoas com produtos, procedimentos e processos no ambiente da assistência a saúde. Um erro de utilização em uma bomba de infusão pode ter consequências graves. Pode-se argumentar que erros nessas circunstâncias podem ser evitados por profissionais com mais informações sobre o desfecho de erros da bomba de infusão que está utilizando. A proposta de aplicação de um instrumento de identificação de conhecimentos para habilidades e usabilidade de bombas de infusão no treinamento do profissional pode reduzir os eventos adversos.

Uma das oportunidades de melhoria desse estudo, além da proposta apresentada, é a sugestão de melhorar o instrumento de notificação da Tecnovigilância da ANVISA da coleta de dados dos eventos adversos relativos a equipamentos médicos, deixando-o mais quantitativo.
$\mathrm{O}$ instrumento apresentado na Figura 1 pode ser utilizado como referência para os fabricantes de BI dentro de seu controle de projeto e novos produtos. $\mathrm{O}$ instrumento também poderá auxiliar na revisão de projetos e gerenciamento de risco para melhorias de produtos.

\section{Referências}

Amoore J, Adamson L. Infusion devices: Characteristics and risk management. Nursing Standard. 2003; 17(28):4552. http://dx.doi.org/10.7748/ns2003.03.17.28.45.c3366

Anderson JG, Jay SJ, Anderson M, Hunt TJ. Evaluating the capability of information technology to prevent adverse drug events: a computer simulation approach. Journal of the American Medical Informatics Association. 2002; 9(5):47990. http://dx.doi.org/10.1197/jamia.M1099

Aspden P, Wolcott J, Bootman JL. Preventing medication errors. Quality Chasm Series. Institute of Medicine of the Nationa Academies. Commitee on Identifying and Preventing Medications Errors. Washington: National Academy Press; 2007.

Associação Brasileira de Normas Técnicas - ABNT. NBR IEC 60601-2-24: 1999: Equipamento eletromédico Parte 2: prescrições particulares para segurança de bombas e controladores de infusão. Rio de Janeiro: ABNT; 1999.

Associação Brasileira de Normas Técnicas - ABNT. NBR ISO 14971: 2009: Produtos para a saúde - aplicação de gerenciamento de risco a produtos para a saúde. Rio de Janeiro: ABNT; 2009.

Associação Brasileira de Normas Técnicas - ABNT. NBR IEC 60601-1-6: 2011: Equipamento eletromédico Parte 1-6: requisitos gerais para segurança básica e desempenho essencial - norma colateral: usabilidade. Rio de Janeiro: ABNT; 2011a.

Associação Brasileira de Normas Técnicas - ABNT. NBR 9241-11: 2011: Requisitos Ergonômicos para Trabalho de Escritórios com Computadores Parte 11 - orientações sobre usabilidade. Rio de Janeiro: ABNT; 2011b.

Baker KN, Flyin EA, Pepper GA, Battes DW, Mikeal RL. Medications errors observed in 36 health care facilities. Archives of Internal Medicine. 2002; 162(16):1897-903. http://dx.doi.org/10.1001/archinte.162.16.1897

Berwick DM, Leape LL. Reducing errors in medicine. BMJ. 1999; 319(7203):136-7. http://dx.doi.org/10.1136/ bmj.319.7203.136

Button VLSN. Dispositivos de infusão. In: Calil JS, organizadores. Equipamentos médico hospitalares e o gerenciamento da manutenção. Brasília: ANVISA; 2002.

Brasil. Agencia Nacional de Vigilância Sanitária - ANVISA. Resolução RDC n 67 de 21 de dezembro de 2009. Dispõe sobre normas de tecnovigilância aplicáveis aos detentores de registro de produtos para saúde no Brasil. Diário Oficial da República Federativa do Brasil, Brasília, dez. 2009.

Brasil. Ministério da Saúde. Secretaria de Ciência, Tecnologia e Insumos Estratégicos. Departamento de Ciência e 
Tecnologia. Diretrizes Metodológicas: Elaboração de estudos para avaliação de equipamentos médicos assistenciais. Brasília, Ministério da Saúde, 2013.

Cybis W. Ergonomia e usabilidade: Conhecimentos, métodos e aplicações. São Paulo: Novatec Editora; 2007.

Food and Drug Administration - FDA. Center for devices and radiological health. U.S. Food and Drug Administration [Internet]. 2010 [cited 2012 Fev 12]. Available from: http://www.fda.gov/ MedicalDevices/ProductsandMedicalProcedures/ GeneralHospitalDevicesandSupplies/InfusionPumps/ ucm202501.htm.

Gawande A. Better: A surgeon's notes on performance. New York: Metropolitan Books; 2007.

Ginsburg G. Human factors engineering: A tool for medical device evaluation in hospital procurement decision-making. Journal of Biomedical Informatics. 2005; 38(3):213-9. http:// dx.doi.org/10.1016/j.jbi.2004.11.008

Kushnituk AW, Patel VL. Cognitive and usability engineering methods for the evaluation of clinical information systems. Journal of Biomedical Informatics. 2004; 37(1):56-76. http:// dx.doi.org/10.1016/j.jbi.2004.01.003

Guimarães LBM. Ergonomia cognitiva: processamento da informação, IHC, engenharia de sistemas cognitivos, erro humano. Editado por Lia Buarque de Macedo Guimarães. 3. ed. Porto Alegre: FEEG/UFRGS; 2006. (Série monográgica ergonomia).

Lima W. Competência: distintas abordagens e implicações na formação de profissionais de saúde. Interface. 2005; 9(17):369-79. http://dx.doi.org/10.1590/ S1414-32832005000200012

Lopes CD, Lopes FFP. Do risco à qualidade: A vigilância sanitária nos serviços de saúde. Brasília: Anvisa; 2008.

Morita PP. Desenvolvimento de um guia para investigação de incidentes em ambientes de saúde baseado na estrutura de gerenciamento de projetos [dissertação]. Campinas: Universidade Estadual de Campinas; 2009.

Rothschild JM, Keohane CA, Cook EF, Orav EJ, Burdick E, Thompson S, Hayes J, Bates DW. A controlled trial of smart infusion pumps to improve medication safety in critically ill patients. Critical Care Medicine. 2005; 33(3):533-40. http://dx.doi.org/10.1097/01.CCM.0000155912.73313.CD

Runciman WB, Merry AF, Tito F. Error, blame and the law in health care: an antipodean perspective. Annals of International Medicine. 2002; 138(2):974-9.

Saupe R, Cutolo LRA, Wendhausen ALP, Benito GAV. Competência dos profissionais da saúde para o trabalho interdisciplinar. Interface: Comunicação, Saúde e Educação. 2005; 9(18):521-36. http://dx.doi.org/10.1590/ S1414-32832005000300005

Schneider, PJ. Applying human factors in improving medication-use safety. American Journal of Health-System Pharmacy. 2002; 59(12):1155-9.

Torelly EMS. Avaliação de efetividade, custos e eventos adversos de bombas de infusão de medicamentos: um ensaio clínico randomizado [dissertação]. Porto Alegre: Universidade Federal do Rio Grande do Sul; 2009.

Vidal, MC, Carvalho PV. Ergonomia cognitiva: raciocínio e decisão no trabalho. Rio de Janeiro: Virtual Científica; 2008.

Zhang J, Johnson TR, Patel VL, Paige DL, Kubose T. Using usability heuristics to evaluate patient safety of medical devices. Journal of Biomedical Informatics. 2003; 36(1-2):2330. http://dx.doi.org/10.1016/S1532-0464(03)00060-1

Watcher RM. Compreendendo a segurança do paciente. Porto Alegre: Artmed; 2010.

Wears RL, Perry SJ. Human factors and ergonomics in the emergency department. Annals of Emergency Medicine. 2002; 40:206-12. http://dx.doi.org/10.1067/ mem.2002.124900

\section{Autores \\ Léria Rosane Holsbach*, Francisco Jose Kliemann Neto \\ Programa de Pós-graduação em Engenharia de Produção, Universidade Federal do Rio Grande do Sul - UFRGS, Av. Osvaldo Aranha, 99, $5^{\circ}$ andar, CEP 90035-190, Porto Alegre, RS, Brasil.}

\section{Nicole Holsbach}

Centro de Referência e Tecnologias e Insumos Estratégicos em Saúde - CRETIES, Programa de Pós-graduação em Engenharia de Produção, Universidade Federal do Rio Grande do Sul - UFRGS, Av. Osvaldo Aranha, 99, $5^{\circ}$ andar, CEP 90035-190, Porto Alegre, RS, Brasil. 